\begin{tabular}{|c|c|c|c|}
\hline & $\begin{array}{l}\text { Escola Superior } \\
\text { de Cestäooe } \\
\text { Tecnologia } \\
\text { [ipSantarém] }\end{array}$ & 䀦宿 & $\begin{array}{l}\text { ISSN 2029-7564 (online) } \\
\text { SOCIALINĖS TECHNOLOGIJOS } \\
\text { SOCIAL TECHNOLOGIES } \\
2014,4(2) \text {, p. } 285-291\end{array}$ \\
\hline
\end{tabular}

\title{
FULL PARALLAX INTEGRAL 3D DISPLAY AND IMAGE PROCESSING TECHNIQUES
}

\author{
Byung-Gook Lee \\ Dongseo University, Korea, lbg@dongseo.ac.kr \\ Seokmin Hong \\ Dongseo University, Korea, tommyhsm@gmail.com \\ Han Yuan Dong \\ Dongseo University, Korea, dsu.kavin@gmail.com \\ Donghak Shin \\ Dongseo University, Korea, shindh2@gmail.com \\ doi:10.13165/ST-14-4-2-03
}

\section{Abstract}

Purpose - Full parallax integral 3D display is one of the promising future displays that provide different perspectives according to viewing direction. In this paper, the authors review the recent integral $3 D$ display and image processing techniques for improving the performance, such as viewing resolution, viewing angle, etc.

Design/methodology/approach - Firstly, to improve the viewing resolution of $3 D$ images in the integral imaging display with lenslet array, the authors present $3 D$ integral imaging display with focused mode using the time-multiplexed display. Compared with the original integral imaging with focused mode, the authors use the electrical masks and the corresponding elemental image set. In this system, the authors can generate the resolution-improved $3 D$ images with the $n \times n$ pixels from each lenslet by using $n \times n$ time-multiplexed display. Secondly, a new image processing technique related to the elemental image generation for $3 D$ scenes is presented. With the information provided by the Kinect device, the array of elemental images for an integral imaging display is generated. 
Findings - From their first work, the authors improved the resolution of $3 D$ images by using the time-multiplexing technique through the demonstration of the 24 inch integral imaging system. Authors' method can be applied to a practical application. Next, the proposed method with the Kinect device can gain a competitive advantage over other methods for the capture of integral images of big $3 D$ scenes. The main advantage of fusing the Kinect and the integral imaging concepts is the acquisition speed, and the small amount of handled data.

Originality / Value - In this paper, the authors review their recent methods related to integral 3D display and image processing technique.

Keywords - integral imaging, lenslet array, elemental images, Kinect.

Research type-general review.

\section{Introduction}

Until now, many techniques for the display of 3D images have been studied (Lippmann, 1908; Okano, et al., 1997; Lee, et al., 2001; Shin, et al., 2005; Jang, et al., 2003; Jin, et al., 2004). The 3D display can be largely classified into two different methods: the stereoscopic display and the real 3D display. Stereoscopic methods provide the user with two different images of the $3 \mathrm{D}$ scene. The images are shown independently to the eyes of the observer so that they produce binocular disparity. This method has been conventionally implemented by the use of special glasses. However, the main drawback of stereoscopy is that it produces ocular fatigue due to the conflict between convergence and accommodation.

On the other hand, the real 3D displays can provide different perspectives when the observer displaces parallel to the display. There are many kinds of the real 3D display methods, including multi-view methods, holographic method and integral imaging methods. Among them, integral imaging reconstructs any point of the 3D scene through the intersection of many rays, and thus, it provides the observer with full-parallax images. Its main advantage is to be implemented with the available $2 \mathrm{D}$ imaging and display technology, such as charge-coupled device (CCD) sensors and LCD displays. However, there are still some physical limitations, including the poor viewing angle, technological hardware limitations and a wider transmission bandwidth of integral images. However, the authors of this paper expect that these limitations will be overcome in the next few years.

\section{Theoretical background}

Integral imaging is a 3D image display technique with full parallax and continuous viewing points. It has been the subject of much research (Lippmann, 1908; Okano, et al., 1997; Lee, et al., 2001; Shin, et al., 2005; Jang, et al., 2003; Jin, et al., 2004). In the pickup part of integral imaging, the rays coming from $3 \mathrm{D}$ objects are recorded as $2 \mathrm{D}$ elemental images with a lenslet array (or pinhole array) and a $2 \mathrm{D}$ image sensor. In the display part 
of II, the recorded 2D elemental images are displayed on a display device, and rays coming from the elemental images are then gathered in space to form 3D real images.

Recently, studies have been reported for two kinds of integral imaging according to the gap distance between a lenslet array and a display panel; depth-priority integral imaging (DPII) and resolution-priority integral imaging (RPII) (Jang, et al., 2003; Jin, et al., 2004). RPII is obtained when the gap distance is not equal to the focal length of the lenslets. It gives 3D images with high resolution and small depth. On the other hand, DPII is obtained by setting the gap equal to the focal length of the lenslets. It provides 3D images with low resolution and large depth through both real and virtual image fields.

\section{Research methodology}

In this paper, the authors first review their resolution improvement method of 3D image in DPII using time-multiplexed electrical mask. While the conventional DPII system generates 3D images with the number of lenslets due to observation of a single pixel from each lenslet, the proposed system can generate $3 \mathrm{D}$ images with the $n \times n$ pixels from each lenslet by using time-multiplexed electrical masks and elemental images.

The proposed DPII system is shown in Fig. 1(a). The gap between the lens array and the display panel is $f$, which is the same as the focal length of single lenslet. A transparent LCD display is located in front of lenslet array to display the mask pattern electrically. Mask patterns are white black checker board, as shown in the center of Fig. 1(a). Figure 1 (a) shows an example of $2 \times 2$ time-multiplexed display using mask patterns. For each lenslet with the size of $d$, one white block and three black blocks are matched.

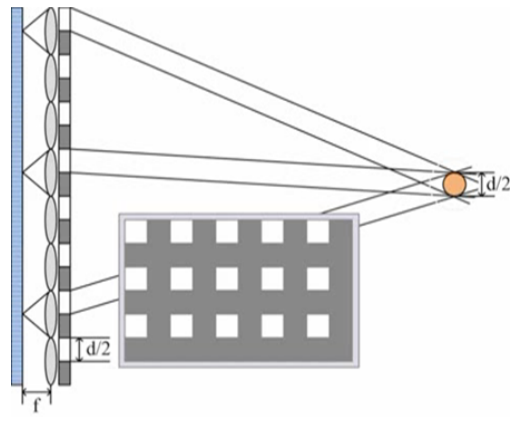

(a)

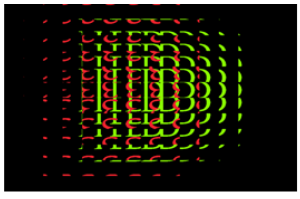

$<\mathrm{x} 1, \mathrm{y} 1>$

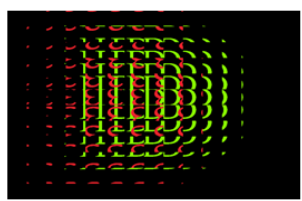

$<\mathrm{x} 2, \mathrm{y} 1>$

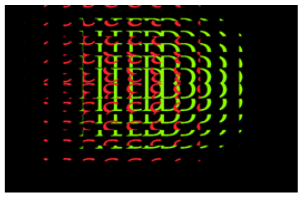

$<\mathrm{x} 1, \mathrm{y} 2>$

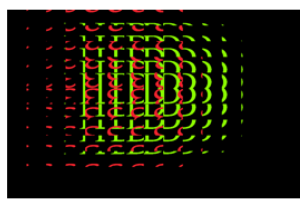

$<\mathrm{x} 2, \mathrm{y} 2>$

(b)

Figure 1. (a) Proposed DPII system, (b) Synthesized 4 elemental images.

4 elemental images for corresponding display have to be generated. The authors of this paper modified the synthesis method of elemental images described in Ref. Jang, et al. (2003), by considering the center position of white block. The synthesized elemental images are shown in Fig. 1(b). In the conventional DPII system, a single ray starting from the display panel becomes parallel beam through the lenslet. The size of the integrated beam is the same with lenslet size $d$. In this method, however, the mask pattern can block 
the beam partially and the diameter of the integrated beam can reduce to the size of white block $(d / 2)$ in electrical mask. This implies that the integrated 3D image point can be reduced to the block size $(d / 2)$, and thus, the resolution of $3 \mathrm{D}$ image can be improved by $2 \times 2$ times. If $n \times n$ time-multiplexed electrical masks are used, the resolution of $3 \mathrm{D}$ images can be improved by $n \times n$ times.

Next, the authors present a new image processing technique related to the elemental image generation for $3 \mathrm{D}$ scenes. There are various methods to capture the information of a three-dimensional scene, in this contribution the authors used the Kinect device, which was initially launched by Microsoft as an add-on accessory for the Xbox game console in 2010 (Microsoft Kinect; Shotton, et al., 2011; Kramer, et al., 2012). Its distinctive hallmark of this device is its capability for capturing simultaneously the RGB image and the depth information in real-time through its own two types of cameras, a RGB camera and an infra-red (IR) one. However, there is big limitation that two cameras are physically separated from each other and have different fields of views. To solve these problems, the authors calibrate both cameras to map each other and reassign its mapped RGBD information into each point located in a 3D virtual space. Next, as shown in Fig. 2, point cloud system was concluded based on the Kinect's live scene which is defined by point with six values: its $(x, y, z)$ coordinates and RGB color intensities.

To project point cloud onto an integral imaging monitor, all points are rearranged based on its depth distance to prevent wrong projection result, and Fig. 3 shows it well. The units are converted from pixels to millimeters to display into physical monitor system. Finally, the values of points of the micro images are assigned by back projection through the virtual pinholes.

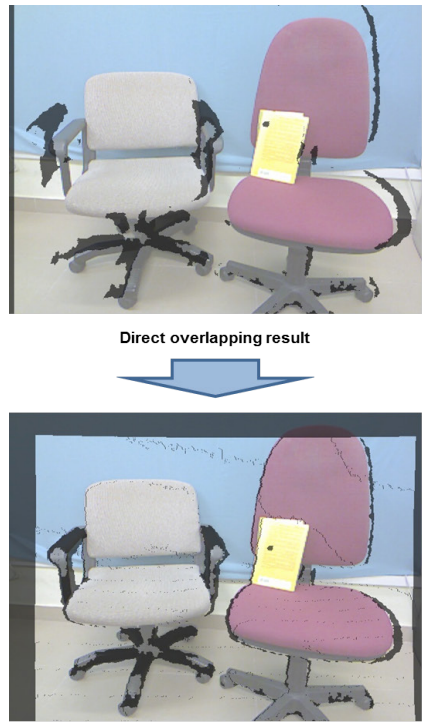

Mapping result
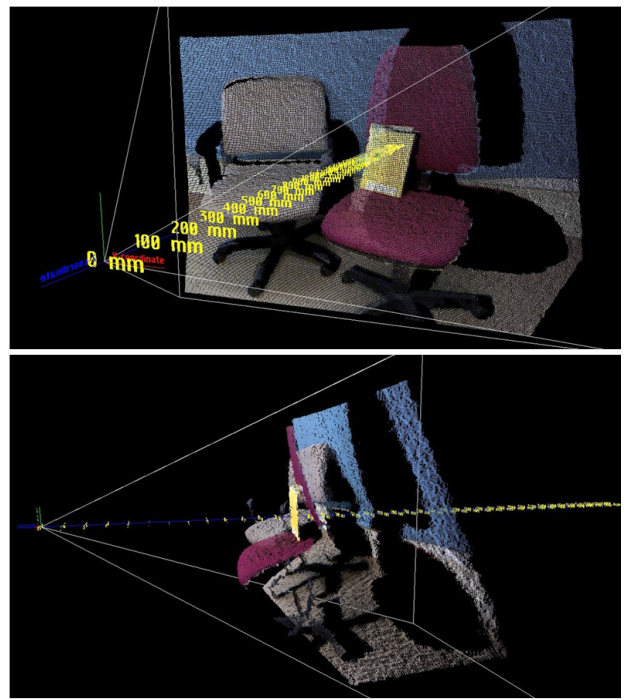

Display 3D point clouds into virtual 3D space

Figure 2. Calibrate two cameras and display 3D point cloud into virtual 3D space 


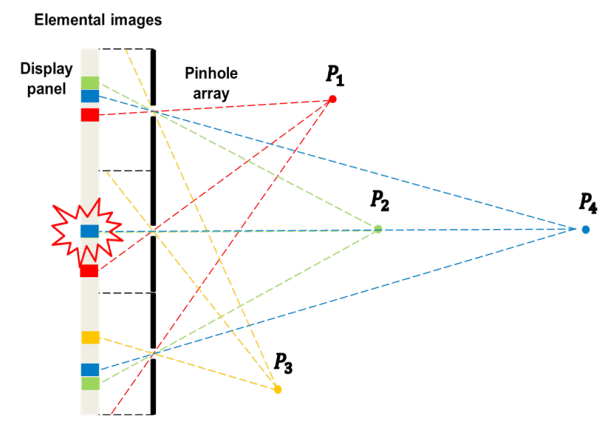

(a)

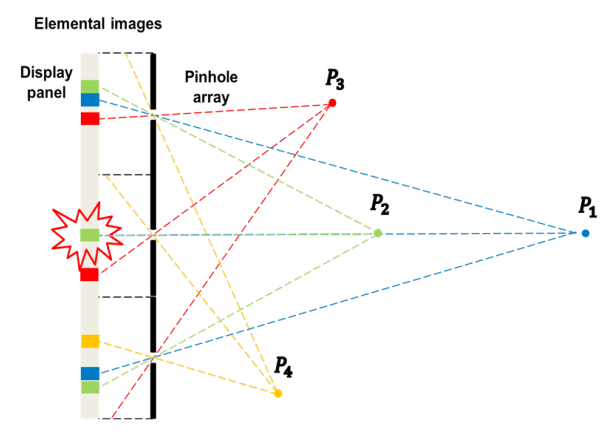

(b)

Figure 3. (a) Occurred wrong projection with non-arranged sequential points, (b) Solve projection problem with rearranged points based on its depth information

\section{Results and findings}

In integral imaging display, to improve the resolution of 3D images displayed in DPII, the authors performed the preliminary experiments. The optical test system was implemented, as shown in Fig. 4(a). The authors used the lenslet array, which consisted of $15 \times 15$ lenslets which focal length and diameter were $10 \mathrm{~mm}$ and $5 \mathrm{~mm}$, respectively. To obtain DPII system, the distance was set between display panel and lenslet array to 10 $\mathrm{mm}$. The elemental images are projected into the rear screen which is used as a display panel. The electrical masks are displayed in transparent LCD. 4 kinds of electrical masks were used to obtain $2 \times 2$ time-multiplexed display. $3 \mathrm{D}$ images are of two characters: ' 3 ' image and 'D' image. ' 3 ' and ' $D$ ' are located at $30 \mathrm{~mm}$ and $-30 \mathrm{~mm}$, respectively. Note that DPII system can display 3D images in real and virtual image field at the same time.

The authors observed the 3D images at the different observation angles in the conventional method and the proposed method. The observed images are shown in Fig. 4. Since $2 \times 2$ time-multiplexed display was used, the proposed method as shown in Fig. 4(c) can improve the resolution of $3 \mathrm{D}$ images by $2 \times 2$ times compared with the conventional method, as shown in Fig. 4(b).

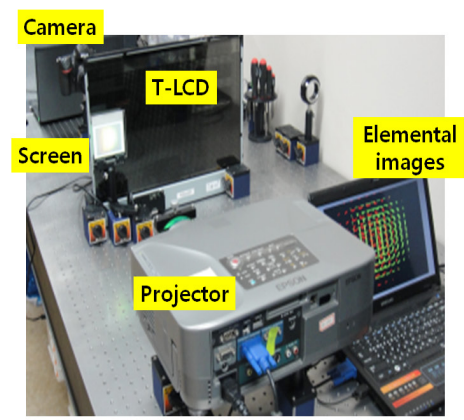

(a)

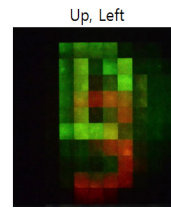

Down, Left

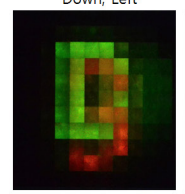

(b)
Up, Right

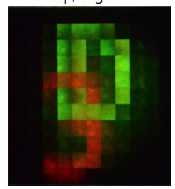

Down, Right

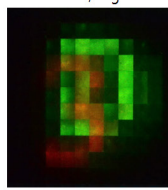

Up, Left

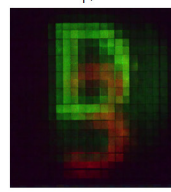

Down, Left

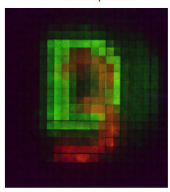

(c)
Up, Right

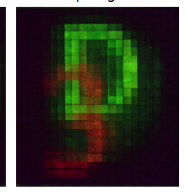

Down, Right

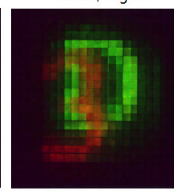

Figure 4. (a) Optical setup, (b) Observed 3D images in the conventional method, (c) Observed 3D images in the proposed method 
Next, to confirm and prove the utility of the approach done, the human model was captured and tested onto the retina display of the iPad. The authors displayed an integral image which is composed with the back projection from point cloud based on the Kinect's view. Note that in this result shown in Fig. 5, the reference plane is set, which is virtual pinhole arrays just middle of two human models. As a result, the authors obtained various horizontal and vertical perspectives with floating in front of the display monitor.

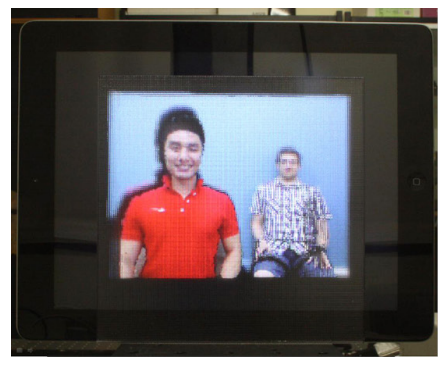

(a)

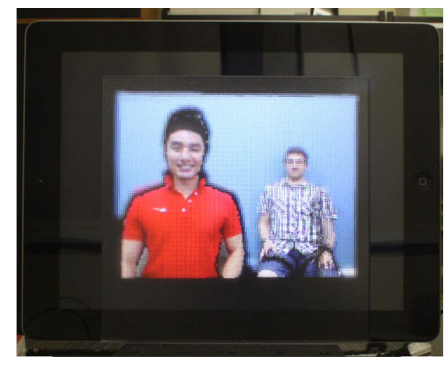

(b)

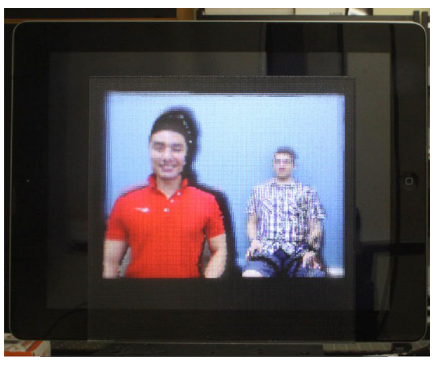

(c)

Figure 5. Obtained different perspectives (a) left, (b) center, (c) right positions from the monitor

\section{Conclusions}

In conclusion, the authors of this paper have presented a review of their previous results for the capture, processing and display of 3D images by using integral imaging technique. The DPII based integral imaging method may be suited for a future real 3D display system. The main advantage of fusing the kinect and the integral imaging concepts is the acquisition speed, and the small amount of handled data. Also, the algorithms proposed are simple. The authors have demonstrated the usefulness of their methods through the optical experiments. In further research, the authors will implement the realtime $3 \mathrm{D}$ display system based on integral imaging for the next-generation $3 \mathrm{D}$ display system.

\section{Acknowledgments}

This work was supported by the IT R\&D program of MKE/KEIT [10041682, Development of high-definition 3D image processing technologies using advanced integral imaging with improved depth range].

\section{Literature}

Jang, J.-S., Jin, F., and Javidi, B. ThreeDimensional Integral Imaging with Large Depth of Focus Using Real and Virtual Image Fields. Opt. Lett. 2003, 28: 1421-1423.
Jin, F., Jang, J.-S., and Javidi, B. Effects of Device Resolution on Three-dimensional Integral Imaging. Opt. Lett. 2004, 29: 1345-1347. 
Kramer, J., Burrus, N., Echtler, F., Daniel, H. C., and Parker, M. Multiple Kinects. In: Hacking the Kinect. Apress, 2012, p. 207-246.

Lee, B., Jung, S.Y., Min, S.-W., and Park, J.-H. Three-dimensional Display by Use of Integral Photography with Dynamically Variable Image Planes. Opt. Lett. 2001, 26: 1481-1482.

Lippmann, G. La photographic integrale. C.R. Acad., Sci. 1908, 146: 446-451.

Microsoft Kinect. [interactive]. <http://www. xbox.com/en-us/kinect/>.

Okano, F., Hoshino, H., Arai, J., and Yuyama, I. Real-time Pickup Method for a Three- dimensional Image Based on Integral Photography. Appl. Opt. 1997, 36: 1598-1603. Shin, D.-H., Cho, M., and Kim, E.-S. Computational Implementation of Asymmetric Integral Imaging by Use of Two Crossed Lenticular Sheets. ETRI J. 2005, 27: 289-293.

Shotton, J., Fitzgibbon, A., Cook, M., Sharp, T., Finocchio, M., Moore, R., et al. Real-time Human Pose Recognition in Parts from Single Depth Images. In Proceedings of the IEEE Computer Society Conference on Computer Vision and Pattern Recognition. 2011. 